\title{
WHICH IS YOUR DIAGNOSIS?
}

Marcelo Souto Nacif ${ }^{1,4}$, Amarino Carvalho de Oliveira Júnior ${ }^{2,4}$, Denise Madeira Moreira ${ }^{3,4}$, Mônica Regina Nagano ${ }^{4}$, José Hugo Mendes Luz ${ }^{4}$, Marcio dos Santos Martinss ${ }^{4}$, Mauro Esteves de Oliveira ${ }^{4}$, Luiz Fernando Mendes $^{4}$, Marcelo Montera ${ }^{5}$, Carlos Eduardo Rochitte ${ }^{6}$

Study developed at Hospital Pró-Cardíaco, Rio de Janeiro, RJ, Brazil. 1. Professor at Faculdade de Medicina de Teresópolis (Unifeso), Teresópolis, RJ, Post-graduation Sub-coordinador at Instituto de Pós-Graduação Médica Carlos Chagas (IPGMCC), Doctoral Fellow in Radiology (Cardiac Magnetic Resonance Imaging) by Universidade Federal do Rio de Janeiro (UFRJ), Rio de Janeiro, RJ, Brazil. 2. Coordinator for the Service of Radiology and Imaging Diagnosis (SRDI) at Hospital Pró-Cardíaco, Rio de Janeiro, RJ, Brazil. 3. Doctor Professor (Adjunct) at Universidade Federal do Rio de Janeiro (UFRJ), Rio de Janeiro, RJ, Brazil. 4. MDs., Radiologists at the Service of Radiolog and Imaging Diagnosis (SRDI) - Hospital Pró-Cardíaco, Rio de Janeiro, RJ, Brazil. 5. MD, Cardiologist at Hospital Pró-Cardíaco, Rio de Janeiro, RJ, Brazil 6. Professor, Private Docent at Universidade de São Paulo, São Paulo, SP, Advisor for Hospital Pró-Cardíaco, Rio de Janeiro, RJ, Brazil. Mailing address: Prof. Dr. Marcelo Souto Nacif. Rua Tavares de Macedo, 136, ap. 1503/A, Icaraí. Niterói, RJ, Brazil, 24220-211. E-mail: msnacif@yahoo.com.br

A male, 27-year old patient weighting $78 \mathrm{~kg}$, with $1.77 \mathrm{~m}$ in height, presenting frequent ventricular arrhythmias has been referred to the Service of Radiology and Imaging Diagnosis of Hospital Pró-Cardíaco to be submitted to cardiac magnetic resonance imaging $(\mathrm{MRI})$.

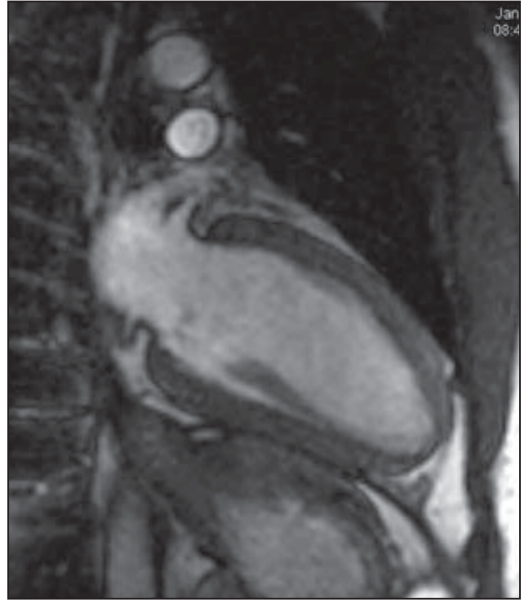

A

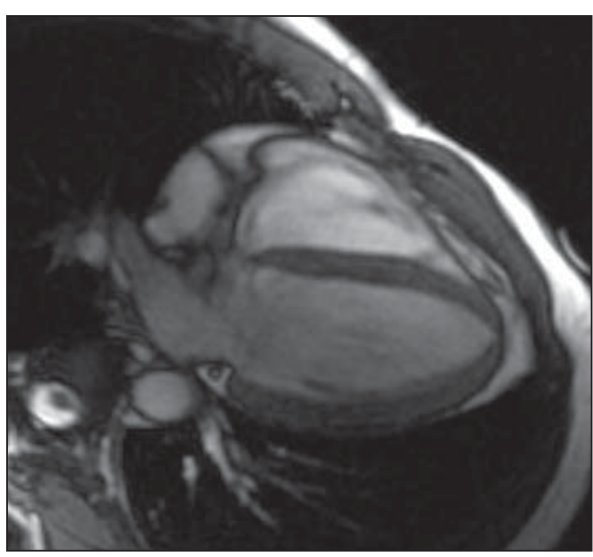

B

Figure 1. Images acquisition with ECG-gating, in cine-Fiesta (SSFP) sequences, at end-diastole, twochamber $(\mathbf{A})$ and four-chamber $(\mathbf{B})$ long axis.

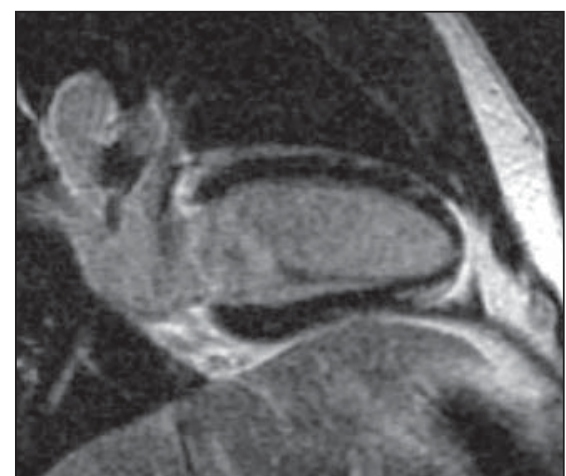

A

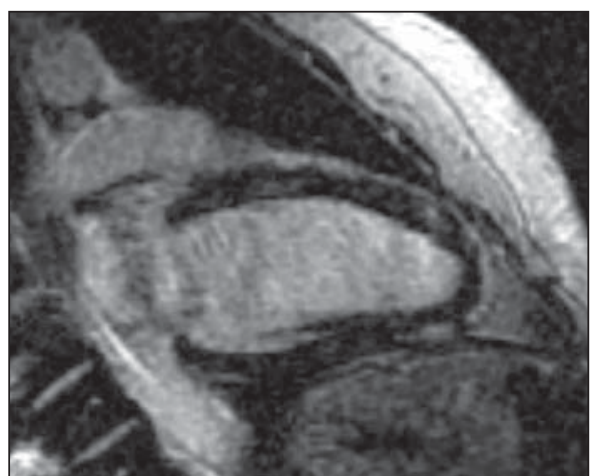

B

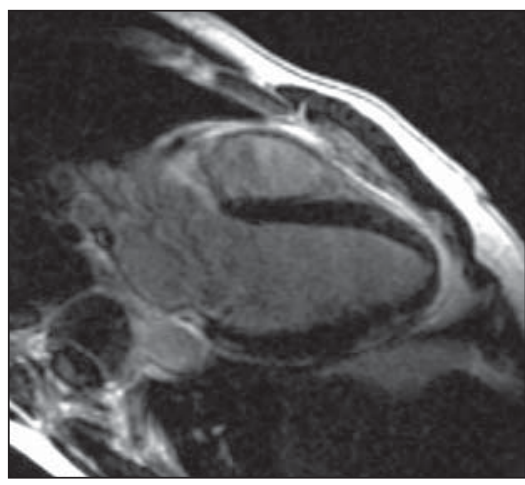

C

Figure 2. Images acquisition with ECG-gating. Delayed enhancement, two-chamber long axis (A,B), and left ventricular outflow tract plane (C). 


\section{Images description}

Figure 1. Images acquisition with electrocardiographic (ECG) gating, in cine-Fiesta (SSFP) sequences, at end-diastole, two-chamber (A) and four-chamber (B) long axis. Note that cardiac chambers, atria and ventricles present with preserved diameters; LV global and segmental functions were preserved.

Figure 2. Images acquisition with ECG-gating. Delayed enhancement on two-chamber, long axis (A,B), and left ventricle outflow tract plane $(\mathrm{C})$. Note the presence of foci and areas of delayed myocardial enhancement in mesocardial and epicardial regions, compatible with the presence of a non-territorial myocardial lesion, i.e. coronary segments are not involved.

Diagnosis: Viral myocarditis.

\section{COMMENTS}

Myocarditis is characterized by the presence of an inflammatory response resulting from several forms of injury. In association with myocarditis, the inflammatory process may eventually affect other heart structures, causing pericarditis or coronary vasculitis.

The majority of forms of myocardial injury are secondary to infection that may have viruses, bacteria, fungi and protozoans as causative agents. However, it is important to observe that several systemic diseases or autoimmunemediated injuries may account for myocarditis, such as peripartum cardiomyopathies or those caused by radiation therapy or chemotherapy.

Amongst the most frequent infectious agents are viruses, especially the Coxackie virus B of the enterovirus family, accounting for about $50 \%$ of cases. Most recently, however, herpes virus and parvovirus B19 have been reported as relevant causative infectious agents for myocarditis.

Myocarditis secondary to viral injury clearly presents three different phases of injury and inflammatory response.

The first phase is characterized the presence of viremia with myocardial viral infection. In this phase there is a viral invasion into cardiomyocytes, promot- ing a direct injury and the resulting immunological response mediated by local tissue macrophages and lymphocytes, the so called T-killers. This local cellular activation promotes an increase in the cytokine mRNA expression [eg. tumor necrosis factor (TNF-alpha), interleukin1 (IL-1) and gamma-interferon (IFN-g) $]^{(\mathbf{1 , 2})}$.

In this initial phase of the disease, the utilization of prednisolone is associated with an increase in the viral titer and decrease in the levels of antibody 6 , which demonstrates the significance of recognizing it in the clinical practice. Other mediators such as perfurin are release by lymphocytes in order to fight the virus and end up injuring the cardiomyocyte. The peak of the first phase occurs between the fifth and seventh day of the disease progression ${ }^{(2,3)}$.

The second phase is marked by the activation of the cellular-immune response resulting from the exposure of viral peptides on the cardiomyocyte surface in histocompatibility complexes that will be recognized by t-lymphocytes. Tlymphocytes play a significant role limiting the viral replication in the destruction of infected cardiomyocytes, which is clearly demonstrated in animal models $^{(1,3,4)}$

In this phase, B lymphocytes represent about $10 \%$ to $20 \%$ of the cellularity with a later progressive increase at the end of a three-month period ${ }^{(5)}$.

The third phase presents three different models of progression. Autoantibodies-mediated progression of the attack to cardiomyocytes may occur. This autoimmune injury is mediated by the primary viral attack or, most frequently, by the persistence of the virus in the myocardium, which may occur in up to $40 \%$ of patients ${ }^{(1,3,5)}$

This permanent autoimmune-mediated injury leads to a progressive myocardial function loss, with development of dilated cardiomyopathy and progressive dysfunction. In this chronic, active presentation of myocarditis there is an abnormal expression of human leukocyte antigen (HLA) DR in the myocardial tissue, and circulating autoantibodies, with absence of cellular infiltrate and myocytolysis $^{(2,5)}$.
Another model of progression is the development of dilated cardiomyopathy as result of ventricular remodeling, where the dysfunction degree is stable. And, finally, the inflammatory process may resolve with recovery of the ventricular function or stabilize with mild ventricular dysfunction ${ }^{(2,5)}$.

In the clinical practice, it is essential, for the doctors, to identify the phase of myocarditis progression to define both the expectations in relation to diagnostic methods and the therapeutic strategy to be adopted.

\section{Clinical presentation}

Myocarditis clinical presentation is variable, and may be asymptomatic, presenting frequent arrhythmias, sudden death, asymptomatic or symptomatic ventricular dysfunction, and fulminant myocarditis. Asymptomatic ventricular dysfunction resolves in $70 \%$ of cases. In symptomatic cases, about $25 \%$ resolve, $50 \%$ stabilize, and $25 \%$ present progression with worsening of the ventricular function ${ }^{(\mathbf{1}, \mathbf{2}, \mathbf{4})}$.

The hypothesis of myocarditis should be considered in the presence of a previous history of: 1) recent onset of ventricular systolic dysfunction (LV or both) with or without symptoms of cardiac failure; 2) progressive ventricular dysfunction with no determining factor; 3) ventricular dysfunction associated with infectious respiratory syndromes or immunological diseases; 4) significant, spontaneous improvement in ventricular dysfunction or dilatation with no pharmacological justification; 5) presence of frequent ventricular arrhythmias, in the absence of a determining factor other than the inflammatory factor; 6) aborted sudden death in young patients.

\section{Magnetic resonance imaging}

Gadolinium-enhanced magnetic resonance imaging (MRI) demonstrates the presence of insterstitial alteration resulting from inflammatory process or ischemic injury.

T1- and T2-weighted sequences with fat-suppression may be of help in the detection of myocardial edema resulting from inflammatory process ${ }^{(\mathbf{5 , 6})}$. 


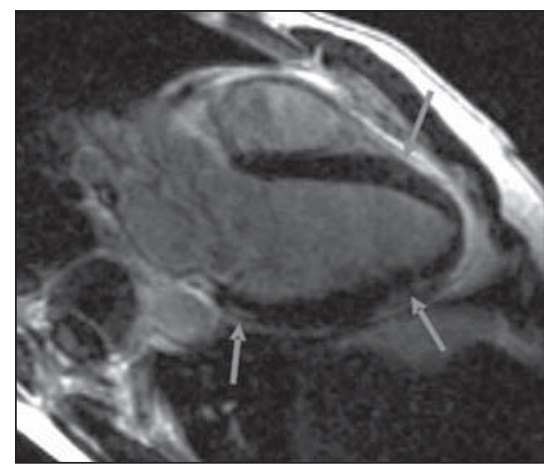

A

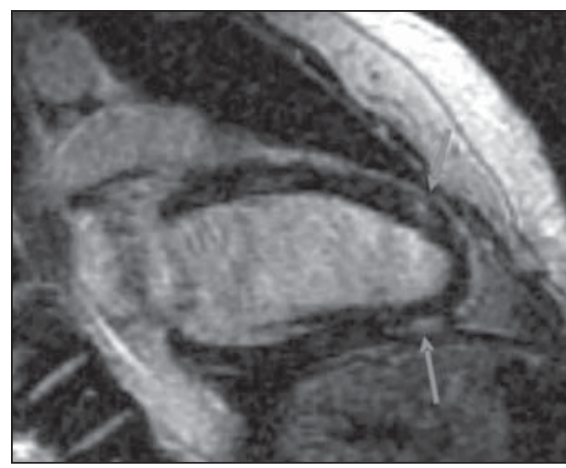

B

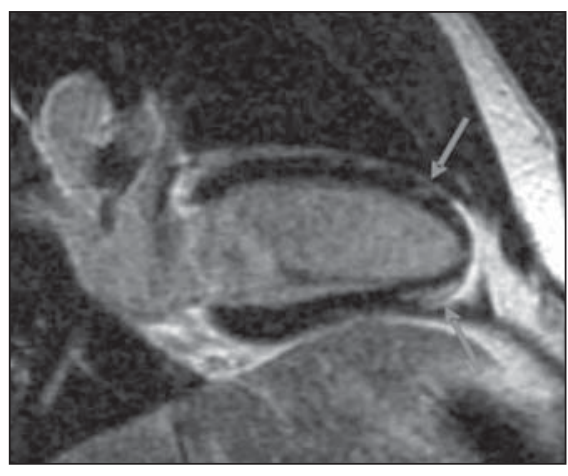

C

Figure 3. Images acquisition with ECG-gating. Delayed enhancement on two chamber, long axis views (A,B) and left ventricle outflow tract plane (C). Note the presence of delayed enhancement foci and areas (arrows) in mesocardial and epicardial regions, always without involvement of the endocardial border (site of coronary disease) in inferolateral basal, anterior and inferoapical, anterolateral medial, lateral and septoapical segments, compatible with the presence of myocardial lesion.

In cases of myocarditis, the presence of delayed myocardial enhancement (Figure 3) may demonstrate necrosis or focal inflammatory lesion, most frequently affecting the mesocardial and epicardial portions of the lateral wall. These findings are significant, considering that myocardial ischemia resulting from coronary artery disease tends to occur in the subendocardial portion, and for this reason it may be differentiated from myocarditis.

There are only two studies in the literature comparing endomyocardial biopsy findings in patients suspected of having myocarditis with gadolinium uptake; other studies do not present histopathological evidence of myocarditis. Mahrholdt et al. have studied patients with preserved ventricular function and disease progression period of up to eight weeks, and, based on the finding of positive delayed enhancement, demonstrated myocarditis in 19 of 21 patients. These patients were submitted to biopsy in the gadolinium-enhanced region of the left ventricle studied by MRI, which has increased the rate of true-positive results.
This study is aimed at demonstrating the feasibility of utilizing MRI as guidance to indicate the site for endomyocardial biopsy, increasing the accuracy in the investigation of myocarditis ${ }^{(4-6)}$.

Although the presence of a late myocardial enhancement confirms myocarditis, it should be noted that its absence does not exclude this diagnosis. Some cases may just present with edema, and not with myocardial necrosis that is important in the diagnosis by MRI. For this reason, T2-weighted sequences may be utilized for detecting myocardial edema with the aim to increase the sensitivity of the method for this diagnosis. Therefore, late enhancement is specific for myocarditis, but usually with low sensitivity.

\section{Final considerations}

Like scintigraphy, MRI presents higher diagnostic at initial phases of myocarditis (phases 1 and 2), and in later phases of the disease alterations may be related not to the myocardial inflammation, but to the presence of fibrosis. MRI also can be utilized for following-up myocarditis involution.

\section{REFERENCES}

1. Kühl U, Lauer B, Souvatzoglu M, Vosberg H, Schultheiss HP. Antimyosin scintigraphy and immunohistologic analysis of endomyocardial biopsy in patients with clinically suspected myocarditis - evidence of myocardial cell damage and inflammation in the absence of histologic signs of myocarditis. J Am Coll Cardiol 1998;32:13711376.

2. Laissy JP, Messin B, Varenne O, et al. MRI of acute myocarditis: a comprehensive approach based on various imaging sequences. Chest 2002; 122:1638-1648.

3. Roditi GH, Hartnell GG, Cohen MC. MRI changes in myocarditis - evaluation with spin echo, cine MR angiography and contrast enhanced spin echo imaging. Clin Radiol 2000;55:752-758.

4. Mahrholdt H, Goedecke C, Wagner A, et al. Cardiovascular magnetic resonance assessment of human myocarditis. A comparison to histology and molecular pathology. Circulation 2004;109:12501258.

5. Friedrich MG, Strohm O, Schulz-Menger J, Marciniak H, Luft FC, Dietz R. Contrast media-enhanced magnetic resonance imaging visualizes myocardial changes in the course of viral myocarditis. Circulation 1998;97:1802-1809.

6. Stillman AE, White RD. Adult heart disease excluding myocardial ischemia and viability. In: Edelman RR, Hesselink JR, Zlatkin MB, Crues $\mathrm{JV}$, editors. Clinical magnetic resonance imaging. 3rd ed. Philadelphia, PA: Saunders-Elsevier, 2006; 1016-1040. 